\title{
Plantes aromatiques du Plateau des Cataractes (Bassin du Congo). Caractérisation du chémotype de l'huile essentielle de Cymbopogon nardus (L.) Rendle acclimaté au Congo-Brazzaville
}

\author{
Thomas Silou ${ }^{(1)}$, Raphaël Bikanga ${ }^{(2)}$, Samuel Nsikabaka ${ }^{(1)}$, Jayneureuse Nombault ${ }^{(1)}$, \\ Chancelvie Mavoungou ${ }^{(1)}$, Gilles Figuérédo ${ }^{(3)}$, Jean-Claude Chalchat ${ }^{(4)}$ \\ (1) Université Marien Ngouabi. Faculté des Sciences et Techniques. École Supérieure de Technologie des Cataractes. BP 389. \\ Brazzaville (Congo).E-mail : thsilou@yahoo.fr \\ (2) Université des Sciences et Techniques de Masuku. Faculté des Sciences. BP 901. Franceville (Gabon). \\ (3) LEXVA Analytique. 7, Rue Henri Mondor. Biopôle Clermont-Limagne. FR-63360 Saint Beauzire (France). \\ (4) AVAHEA. 38, avenue de Clémensat. FR-63540 Romagnat (France).
}

Reçu le 4 novembre 2016, accepté le 5 mai 2017.

Cet article est distribué suivant les termes et les conditions de la licence CC-BY (http://creativecommons.org/licenses/by/4.0/ deed.fr)

Description. Les huiles essentielles de Cymbopogon nardus (L.) Rendle, Cymbopogon winterianus Jowitt, Cymbopogon flexuosus (Nees ex Steud.) Wats et Cymbopogon citratus L. (DC.) Stapf (citronnelle) sont constituées à plus de $80 \%$ de citronellal, géraniol, citral, citronellol, acétate de géranyle et limonène. Elles ne se distinguent les unes des autres que par les proportions relatives de ces différents constituants. Il est donc important de connaitre la composition chimique exacte des huiles essentielles produites.

Objectifs. Évaluer le chémotype de C.nardus acclimaté sur le Plateau des Cataractes.

Méthode. Les huiles essentielles de C. nardus acclimatées sur le Plateau des Cataractes ont été extraites par hydrodistillation et analysées par chromatographie (GC et CG/MS) pendant plus d'une décennie.

Résultats. Avec un profil caractérisé par la composition suivante : citronellal (40-48\%), géraniol (10-22 \%), citronellol (10-12\%), limonène (2-3\%), acétate de géranyle (1-2\%), linalol (1\%), cette espèce produit une huile essentielle de citronella type Java. Ceci tient au fait que la différence entre les types Java et Ceylan de citronella repose uniquement sur les teneurs individuelles de leurs trois principaux constituants : citronellal, géraniol et citronellol. Cymbopogon nardus est connu pour sa variabilité élevée, avec deux variétés et sept sous-variétés.

Conclusions. Le citronella diffusé en Afrique de l'Ouest et du Centre sous le nom de Cymbopogon nardus type Java serait plutôt proche de Cymbopogon winterianus Jowitt par sa composition chimique.

Mots-clés. Cymbopogon, composition chimique, géraniol, citronellol.

Aromatic plants from the Plateau des Cataractes (Congo Basin). Chemotype characterization of essential oil of Cymbopogon nardus (L.) Rendle acclimatized in Congo-Brazzaville

Description. The essential oils of Cymbopogon nardus (L.) Rendle, Cymbopogon winterianus Jowitt, Cymbopogon flexuosus (Nees ex Steud.) Wats and Cymbopogon citratus L. (DC.) Stapf (lemongrass) consist of more than $80 \%$ of the following constituents: citronellal, geraniol, citral, citronellol, geranyl acetate, and limonene. For the purposes of business transactions, it is therefore important to know the exact chemical composition of the essential oils produced.

Objectives. Chemotype evaluation of C.nardus acclimatized on the Plateau des Cataractes.

Method. The essential oils of C. nardus were extracted by hydrodistillation and analyzed by chromatography (GC and GC/ MS) over a period of more than ten years.

Results. Cymbopogon nardus was found to produce a Java type citronella essential oil (C. winterianus) with the following profile: citronellal (40-48\%), geraniol (10-22\%), citronellol (10-12\%), limonene (2-3\%), geranyl acetate (1-2\%), linalool $(1 \%)$. The difference between these two types of citronella, Java and Ceylon, was based on the relative proportions of their three main constituents: citronellal, geraniol and citronellol. Cymbopogon nardus is known for its high variability, with two varieties and seven subvarieties. 
Conclusions. The citronella established in West and Central Africa under the name of Cymbopogon nardus (Java type) could be considered a Cymbopogon winterianus Jowitt, based on the chemical composition of its essential oil.

Keywords. Cymbopogon, chemical composition, geraniol, citronellol.

\section{INTRODUCTION}

Dans le cours inférieur du fleuve Congo, le Plateau des Cataractes, situé entre les villes de Brazzaville (Congo) et Kinshasa (RD Congo) d'une part et le port de Matadi sur l'Océan atlantique, d'autre part, présente un microclimat très favorable aux arbres fruitiers exotiques (litchi, ramboutan, mangoustan, mangues, etc.) et aux plantes aromatiques (eucalyptus, citronnelle, lippie, etc.).

Au Congo-Brazzaville, un projet gouvernemental ciblant principalement Eucalyptus citriodora, appuyé par l'Organisation Internationale des Bois Tropicaux (OIBT), encadre cette activité en plein essor et qui, à terme, vise l'installation d'entreprises et de coopératives de production d'huiles essentielles (Anonyme, 2010).

Sur la même lancée et à des fins de diversification, des espèces du genre Cymbopogon (lemongrass et citronella) sont en cours de diffusion en milieu paysan, particulièrement sur le Plateau des Cataractes, sur les sites de Mbanza-Ndounga, Loukoko, Kiazi, Loumou, Nkama et Voka.

Ces espèces présentent une grande variabilité morphologique et chimique en Inde ou plus généralement dans le Sud-Est asiatique, où elles sont endémiques (Akhila, 2010).

$\mathrm{Au}$ moins quatre espèces fournissent les huiles essentielles commercialisées sous les appellations génériques de lemongrass et de citronella: Cymbopogon flexuosus (Nees ex Steud.) Wats et Cymbopogon citratus L. (DC.) Stapf pour la première et Cymbopogon nardus (L.) Rendle et Cymbopogon winterianus Jowitt, pour la seconde (Ganjewala, 2009).

Les huiles essentielles extraites de ces espèces ont, pour la plupart, les mêmes constituants majeurs $(>80 \%)$ : citronellal, géraniol, citral, citronellol, acétate de géranyle, limonène; leur différence provient des proportions relatives des constituants de ces huiles, mais aussi de la nature de leurs constituants mineurs (1-4\%) et de leurs constituants à l'état de traces $(<0,1 \%)$. Il arrive même que deux espèces formellement distinctes d'un point de vue morphologique présentent des profils chimiques ambigus. Il est donc important, pour les transactions commerciales, de connaitre la composition chimique exacte des huiles essentielles produites.

Nous avons précédemment mis en évidence, sur le Plateau des Cataractes, l'existence de deux chémotypes pour Cymbopogon flexuosus : un à citral (lemongrass), Cymbopogonflexuosus var. ' flexuosus ', et l'autre citronella-like: Cymbopogon flexuosus var. ' albescens' (Silou et al., 2017a ; Silou et al., 2017b).

Cet article se propose de caractériser le(s) chémotype(s) acclimaté(s) sur le Plateau des Cataractes et en cours de diffusion dans cette région, de façon à mieux cerner la valeur commerciale des huiles essentielles en cours de production. On sait, par ailleurs, qu'au Bengladesh, à côté du profil type de C.nardus, appelé variété ' nardus ', on a identifié un chémotype à géraniol $(75,1 \%)$ et acétate de géranyle $(7,6 \%)$ connu comme variété 'confertiflorus' (Chowdhury et al., 1998) et que The International Plant Names Index (cité par Akhila, 2010), par exemple, a retenu sept sous-variétés pour C.nardus : bombycinus, exaltatus, grandis, lanatus, procerus, refractus, schultzii.

\section{MATÉRIEL ET MÉTHODES}

\subsection{Milieu d'étude}

Les plantations étudiées sont installées au Campus Rural de Loukoko, District de Louingui, Département du Pool sur le Plateau des Cataractes, sur un sol argilosablonneux et sous climat bas-congolais (Silou et al., 2017c).

\subsection{Matériel végétal}

Des champs pilotes paysans on été mis en place à partir des éclats de Cymbopogon nardus acquis du Bénin en marge du projet canadien «création de petites entreprises au Togo/Bénin/Ghana »(Koumaglo, 2004) sur sept sites au Campus Rural de Loukoko, pour un total de 2 ha (toutes les plantations sont conçues sur 0,25 ha avec un écartement de $1 \mathrm{~m} \times 1 \mathrm{~m}$ ). Les plantations des sites de Loukoko-colline (LKc), Sésé colline (SSc) et de Ngamibakou colline (NGc) se trouvent sur des versants de colline; celles du site Nkama-plateau (NKp) sont situées sur un mini-plateau au sommet d'une colline, tandis que celles de Nkama vallée (NKv) et Ntiétié vallée (NTv) sont dans des basfonds.

\subsection{Extraction}

Au laboratoire, les extraits volatils des feuilles (séchées à l'ombre pendant 2-4 jours) ont été obtenus par hydro-

\footnotetext{
${ }^{1}$ www.ipni.org
} 
distillation pendant 2-3 h à l'aide d'un extracteur de type Clevenger. Le condensat est ensuite recueilli dans une ampoule à décanter dans laquelle l'huile essentielle est isolée de la phase aqueuse par extraction à l'éther diéthylique. L'extrait est séché sur sulfate de sodium anhydre. L'huile essentielle est récupérée après évaporation du solvant à l'aide d'un rotavapor.

L'extraction artisanale pilote a été faite dans des distillateurs de 60 et 3001 . Le matériel végétal et l'eau de distillation, contenus dans la même cuve, sont séparés par une tôle perforée; le réfrigérant est constitué d'un tuyau de $2 \mathrm{~cm}$ de diamètre interne, muni d'une chambre de détente et traversant une cuve de 60 ou 3001 , remplie d'eau qui circule à contre-courant du distillat. Le chargement maximal en matériel végétal est de $4 \mathrm{~kg}$ pour le distillateur de 601 et de $30 \mathrm{~kg}$ pour le distillateur de 3001 (Figure 1).

\subsection{Analyse chromatographique}

Chromatographie en Phase Gazeuse (CPG). L'analyse quantitative de l'huile essentielle est réalisée sur un chromatographe en phase gazeuse Agilent, modèle 6890, équipé d'une colonne DB5 MS (20 $\mathrm{m} \times 0,18 \mathrm{~mm} \times 0,18 \mu \mathrm{m})$. La température du four est de $50^{\circ} \mathrm{C}$ pendant $3 \mathrm{~min}$ puis augmente jusqu'à $300^{\circ} \mathrm{C}$ à la vitesse de $10^{\circ} \mathrm{C}$ par minute, celle de l'injecteur est de $280^{\circ} \mathrm{C}$. Cet appareil est équipé d'un Détecteur à Ionisation de Flamme (FID) dihydrogène $\left(40 \mathrm{ml} \cdot \mathrm{min}^{-1}\right) /$ Air $\left(450 \mathrm{ml} \cdot \mathrm{min}^{-1}\right)$. Le débit du gaz vecteur (dihydrogène) est de $1 \mathrm{ml} \cdot \mathrm{min}^{-1}$.

Couplage CPG/Spectrométrie de Masse (SM). L'analyse qualitative est réalisée à l'aide d'un chromatographe en phase gazeuse Agilent, modèle 7890 couplé à un spectrophotomètre de masse Agilent, modèle 5975, équipé d'une colonne DB5 MS (20 $\mathrm{m} \times 0,18 \mathrm{~mm} \times 0,18 \mu \mathrm{m})$. La température du four est de $50{ }^{\circ} \mathrm{C}$ et reste constante pendant 3 min puis a

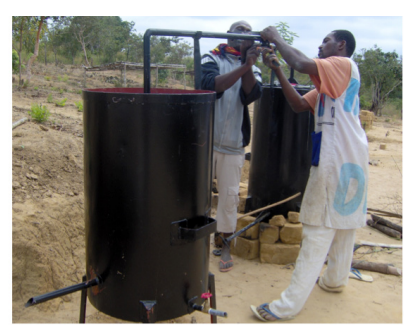

b

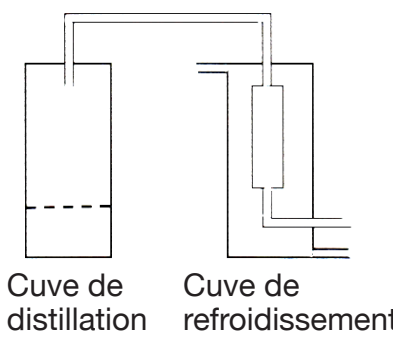

Figure 1. Distillateur $300 \mathrm{~L}$ - The $300 \mathrm{~L}$ distiller.

a. extracteur pilote installé au CRL - pilot distiller installed at $C R L ; \mathbf{b}$. schéma technique - technical diagram. augmente jusqu'à $300^{\circ} \mathrm{C}$ à la vitesse de $8^{\circ} \mathrm{C}$ par minute, celle de l'injecteur est de $280{ }^{\circ} \mathrm{C}$. L'ionisation est faite par impact électronique à $70 \mathrm{eV}$. La température de la source est de $230^{\circ} \mathrm{C}$ et l'échelle de balayage de masse $\mathrm{m} / \mathrm{z}$ varie de 33 à 450 unités. Le débit du gaz vecteur (hélium) est réglé à $0,9 \mathrm{ml} \cdot \mathrm{min}^{-1}$.

L'identification des composés a été réalisée par comparaison de leur spectre de masse et de leur indice de rétention (IR) avec ceux des banques de données NIST (1999), König et al. (2001), Adams (2012) et ceux de notre laboratoire (http//lexva-analytique.com).

\subsection{Traitements statistiques}

La statistique descriptive et les représentations graphiques ont été réalisées sur Excel 8.0 de Microsoft, la statistique multi-variée (Analyse en Composantes Principales, ACP) sur XLSTAT (www.xlstat.com).

\section{RÉSULTATS}

\subsection{Teneur et composition chimique des huiles essentielles de Cymbopogon nardus acclimaté sur le Plateau des Cataractes}

Le tableau 1 donne la composition type d'un échantillon d'huile essentielle de C.nardus acclimaté sur le Plateau des Cataractes et la compare aux profils de citronella type Ceylan (C. nardus) et citronella type Java proche de $C$. winterianus, tels qu'ils apparaissent sur les résultats de la compilation de la littérature (Akhila, 2010).

La figure 2 représente le chromatogramme type de l'huile essentielle de C.nardus, qui est particulièrement simple avec une dizaine de pic à plus de $1 \%$. Quarante-deux constituants ont été détectés et 40 ont été formellement identifiés. Par ordre d'importance décroissant, on trouve: le citronellal $(47,19 \%)$, de très loin le constituant très largement majoritaire, comme dans C.winterianus (30-45\%); le géraniol $(22,32 \%)$, en deuxième position chez C. winterianus, alors qu'il est le premier constituant de C.nardus selon Akhila (2010); le citronellol $(11,98 \%)$, troisième chez C.nardus $(8-20 \%)$ selon Akhila (2010) et chez C. winterianus (4-10 \%). Comme chez $C$. nardus et $C$. winterianus, ces trois constituants représentent plus de $80 \%$ de la composition totale de l'huile étudiée. Quatre constituants ont des teneurs individuelles comprises entre 1 et $4 \%$ : le limonène $(3,18 \%)$, l'élémol $(2,99 \%)$, le béta élémène $(1,19 \%)$, le néo-isopulégol $(1,10 \%)$. Neuf constituants, dont les teneurs sont comprises entre 0,5-1\%, peuvent être considérés comme mineurs : le delta cadinène $(0,93 \%)$, l'acétate de géranyle $(0,73 \%)$, le géranial $(0,69 \%)$, l'alpha cadinol $(0,68 \%)$, l'eugénol $(0,68 \%)$, 
Tableau 1. Composition chimique et teneur des huiles essentielles de Cymbopogon nardus acclimaté sur le Plateau des Cataractes (CN01/12) comparées aux données de la littérature - Content and chemical composition of essential oils of Cymbopogon nardus acclimatized in the Plateau des Cataractes (CN01/12).

\begin{tabular}{|c|c|c|c|c|c|c|c|}
\hline Pic & $\mathbf{T R}(\min )$ & $\mathbf{I R}_{\mathrm{c}}$ & $\mathbf{I R}_{\text {Litt }}$ & Constituant & Teneur $(\%) *$ & $\operatorname{Teneur}(\%)^{* *}$ & Teneur $(\%)^{* * *}$ \\
\hline 1 & 6.885 & 977 & 981 & 6-méthyl-heptane-5-èn-2-one & 0,04 & - & - \\
\hline 2 & 6.959 & 981 & 988 & myrcène & 0,06 & - & - \\
\hline 3 & 7.687 & 1020 & 1024 & limonène & 3,18 & $9-28$ & - \\
\hline 6 & 8.117 & 1045 & $\mathrm{nr}$ & 2,6-diméthyl-hept-5-èn-1-al & 0,12 & - & - \\
\hline 7 & 8.658 & 1076 & 1086 & terpinolène & 0,05 & - & - \\
\hline 8 & 8.909 & 1090 & 1095 & linalol & 0,67 & 8 & - \\
\hline 9 & 9.077 & 1100 & 1106 & cis-oxyde de rose & 0,03 & - & - \\
\hline 10 & 9.368 & 1120 & 1122 & trans-oxyde de rose & 0,07 & - & - \\
\hline 11 & 9.711 & 1145 & 1144 & néoisopulégol & 1,10 & traces & - \\
\hline 12 & 9.807 & 1151 & 1148 & citronellal & 47,19 & $5-16$ & $30-45$ \\
\hline 14 & 9.876 & 1156 & 1155 & isoisopulégol & 0,48 & traces & - \\
\hline 16 & 10.049 & 1169 & 1167 & néoisoisopulégol & 0,07 & traces & - \\
\hline 17 & 10.397 & 1193 & $\mathrm{nr}$ & NI & 0,02 & - & - \\
\hline 18 & 10.429 & 1196 & 1195 & alpha terpinéol & 0,06 & - & - \\
\hline 19 & 10.575 & 1206 & 1201 & décanal & 0,10 & - & - \\
\hline 21 & 10.894 & 1228 & 1223 & citronellol & 11,98 & $8-20$ & $4-10$ \\
\hline 22 & 11.049 & 1239 & 1235 & néral & 0,48 & & - \\
\hline 23 & 11.253 & 1254 & 1249 & géraniol & 22,32 & $20-30$ & $20-25$ \\
\hline 25 & 11.470 & 1269 & 1264 & géranial & 0,69 & - & - \\
\hline 26 & 11.985 & 1306 & 1312 & acide citronellique & 0,19 & - & - \\
\hline 27 & 12.463 & 1342 & $\mathrm{nr}$ & p-menthane-3,8-diol & 0,23 & - & - \\
\hline 28 & 12.552 & 1349 & 1350 & acétate de citronellyle & 0,66 & traces & 3 \\
\hline 29 & 12.603 & 1352 & 1356 & eugénol & 0,68 & traces & - \\
\hline 31 & 12.925 & 1377 & 1379 & acétate de géranyle & 0,73 & traces & 4 \\
\hline 32 & 13.016 & 1384 & $\mathrm{nr}$ & NI & 0,07 & - & - \\
\hline 33 & 13.055 & 1387 & 1387 & béta bourbonène & 0,04 & - & - \\
\hline 34 & 13.111 & 1391 & 1389 & béta élémène & 1,19 & - & - \\
\hline 35 & 13.519 & 1423 & 1417 & béta caryophyllène & 0,03 & - & - \\
\hline 36 & 13.966 & 1459 & 1452 & alpha humulène & 0,05 & - & - \\
\hline 37 & 14.182 & 1476 & 1478 & gamma muurolène & 0,07 & - & - \\
\hline 38 & 14.274 & 1484 & 1484 & germacrène-D & 0,62 & - & - \\
\hline 39 & 14.464 & 1499 & 1500 & alpha muurolène & 0,26 & - & - \\
\hline 40 & 14.649 & 1515 & 1513 & gamma cadinène & 0,17 & - & - \\
\hline 41 & 14.696 & 1519 & 1522 & delta cadinène & 0,93 & - & - \\
\hline 42 & 14.921 & 1538 & 1537 & alpha cadinène & 0,04 & traces & - \\
\hline 43 & 15.062 & 1550 & 1548 & élémol & 2,99 & - & - \\
\hline 44 & 15.403 & 1579 & 1574 & germacrène-D-4-ol & 0,61 & - & - \\
\hline 45 & 15.781 & 1612 & $\mathrm{nr}$ & dihydrofarnésal & 0,04 & - & - \\
\hline 46 & 16.027 & 1634 & 1630 & gamma eudesmol & 0,16 & - & - \\
\hline
\end{tabular}


Tableau 1 (suite). Composition chimique et teneur des huiles essentielles de Cymbopogon nardus acclimaté sur le Plateau des Cataractes (CN01/12) comparées aux données de la littérature - Content and chemical composition of essential oils of Cymbopogon nardus acclimatized in the Plateau des Cataractes (CNO1/12).

\begin{tabular}{|c|c|c|c|c|c|c|c|}
\hline Pic & $\mathbf{T R}(\min )$ & $\mathbf{I R}_{\mathrm{c}}$ & $\mathbf{I R}_{\text {Litt }}$ & Constituant & Teneur $(\%)^{*}$ & Teneur $(\%)^{* *}$ & Teneur $(\%) * * *$ \\
\hline 47 & 16.134 & 1644 & 1638 & épi-alpha cadinol & 0,10 & - & - \\
\hline 48 & 16.154 & 1646 & 1640 & épi-alpha muurolol & 0,17 & - & - \\
\hline \multirow[t]{2}{*}{49} & 16.288 & 1658 & 1652 & alpha cadinol & 0,68 & - & - \\
\hline & & & & Total & 99,42 & & \\
\hline
\end{tabular}

* : présent travail - present work; ** : compilation de la littérature concernant C.nardus : citronella type Ceylan (Akhila, 2010) - compilation of literature concerning C. nardus: citronella type Ceylon (Akhila, 2010); *** : compilation de la littérature concernant $C$. winterianus : citronella type Java (Akhila, 2010) - compilation of literature concerning $C$. winterianus; citronella type Java (Akhila, 2010) ; NI : non identifié - not identified; $\mathrm{nr}$ : non reporté - not available; TR : temps de retention - retention time ; $\mathrm{IR}_{\mathrm{c}}$ : indice de rétention calculé - retention index (calculated); $\mathrm{IR}_{\mathrm{Litt}}$ : indice de rétention dans la littérature - retention index in the literature.

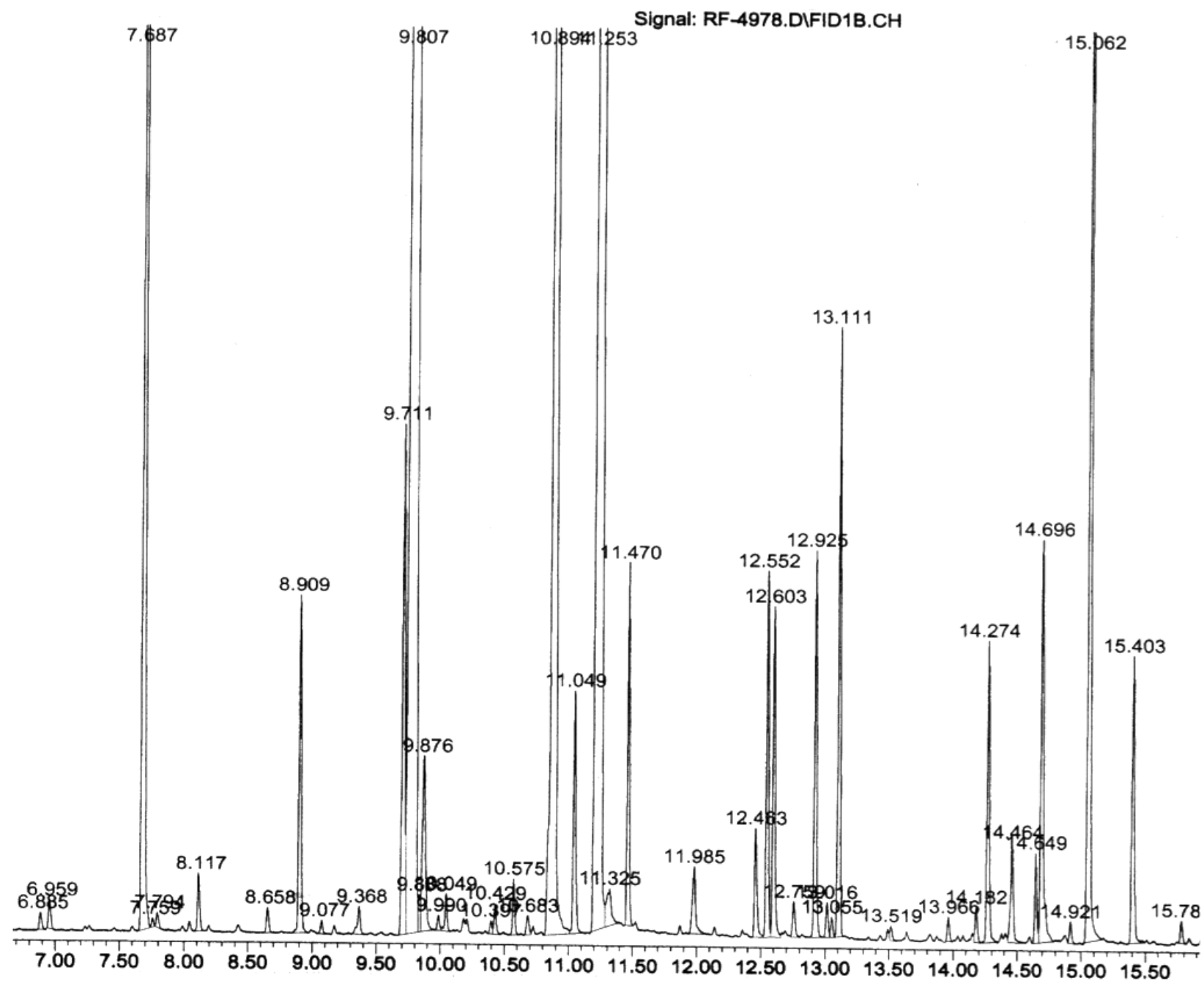

Figure 2. Chromatogramme type de l'huile essentielle de Cymbopogon nardus acclimaté sur le Plateau des Cataractes Chromatogram of the essential oil of Cymbopogon nardus acclimatized in the Plateau des Cataractes. 
le linalol $(0,67 \%)$, l'acétate de citronellyle $(0,66 \%)$, le germacrène $\mathrm{D}(0,62 \%)$ et le germacrène $\mathrm{D}-4-\mathrm{ol}$ $(0,61 \%)$ et 26 constituants, présents à l'état de traces $(<0,5 \%)$, complètent la liste.

\subsection{Variation stationnelle de la teneur et de la composition chimique des huiles essentielles de Cymbopogon nardus acclimaté sur le Plateau des Cataractes}

Des échantillons de sites suffisamment différents ont été analysés afin d'évaluer la variabilité de leurs huiles essentielles. Le tableau 2 regroupe leur composition chimique en constituants majeurs.

Les huiles essentielles analysées présentent toutes le même profil chimique; les faibles valeurs de coefficients de variation $(<15 \%$ pour les constituants majeurs) traduisent une très faible variabilité de la composition chimique des huiles correspondantes.

Afin de mieux visualiser les ressemblances et les différences entre ces huiles, nous avons fait appel à la représentation en coordonnées polaires (radar plots).

La figure 3 représentant des radar plots des huiles essentielles venant de différents sites de Sésé-colline, SSc ; Loukoko-colline, LKc ; Loukoko-vallée, LKv ; Nkama-pltateau, NKp, à titre d'illustration, met en évidence la similitude parfaite de ces huiles quelles que soient les caractéristiques du site : un site en basfonds, deux sur des versants de colline et un sur un mini-plateau au sommet d'une colline.

Le chémotype ( $\%$ citronellal $>\%$ géraniol $>\%$ citronellol $>\%$ limonène $\sim$ élémol) acclimaté sur le Plateau des Cataractes est insensible à « l'effet station ». L'huile essentielle produite a une composition chimique très stable, ce qui est un atout très important sur le plan commercial.

\subsection{Variation annuelle de la composition chimique en constituants majeurs des huiles essentielles de Cymbopogon nardus acclimaté sur le Plateau des Cataractes (campagne 2015)}

Une vingtaine d'échantillons d'huiles essentielles, produites avec le distillateur pilote de $300 \mathrm{~L}$ pendant la campagne 2015, ont été analysés ; la figure 4 illustre la variation de la composition en constituants majeurs et mineurs de ces échantillons. On retrouve les mêmes constituants majeurs (citronellal, géraniol et citronellol) et mineurs pendant toute la campagne (Figure 5).

On note une parfaite stabilité du profil chimique pendant les différentes saisons de l'année agricole.

L'interprétation quantitative des courbes de la figure 4 est moins aisée; nous avons fait appel à la statistique multivariée, l'analyse en composantes principales (ACP), notamment, pour améliorer la lisibilité des résultats. En effet, l'ACP est une méthode de traitement des données contenues dans un tableau de type: Individus (lignes) x Variables quantitatives (colonnes). Elle permet de réduire une grande masse de données en un nombre plus raisonnable et facilement exploitable, en construisant à la place des variables de départ un petit nombre de variables par combinaison linéaire des premières. Ces nouvelles variables sont appelées composantes principales. Elles sont sensées, sous certaines conditions, résumer aussi bien que possible les données de départ. L'ACP est donc une technique de compression des données. Elle permet de visualiser à l'aide de représentations graphiques simples le lien entre les variables et la similitude entre les individus : le cercle de corrélation pour la recherche des liens entre les variables et le plan formé par les deux premières composantes principales $(\mathrm{F} 1, \mathrm{~F} 2)$ pour la recherche des similitudes entre individus (Figure 6).

L'ACP des huiles essentielles étudiées construite sur neuf variables indique l'absence de regroupement en classes des individus dans le premier plan principal (F1, F2) représentant $70 \%$ de l'information sur la variabilité de ces huiles (Tableau 3, Figure 6). Elle indique également que 1 et 2, 3 et 7, 4 et 6 , corrélés deux à deux, sont très bien représentés sur ce plan. Le couple 1 et 2 est anti-corrélé au couple 4 et 6 , tandis que le couple 3 et 7 varie indépendamment de ces deux premiers couples.

En fin de compte, il ressort de cette étude que les teneurs en citronellal et en citronellol ne sont pas liées, alors qu'elles sont anti-corrélées à celle du géraniol, avec des coefficients de corrélation respectifs de $-0,90$ et de -0,60 (Tableau 4).

Ainsi, dans une huile essentielle de citronella donnée, lorsque la teneur en citronellal augmente, celle de géraniol baisse et, à la limite, lorsque leur importance relative s'inverse, on passe d'un chémotype à un autre ; par exemple, du type Java au type Ceylan, et inversement.

\subsection{Variation pluri-annuelle de la composition chimique des constituants majeurs des huiles essentielles de Cymbopogon nardus acclimaté sur le Plateau des Cataractes (2003-2016)}

Par campagne, une dizaine d'échantillons d'huiles essentielles extraites du matériel végétal de 3-4 mois, pris au hasard, ont été analysés. Les résultats des analyses, moyennes par année assorties d'écarts-types, sont regroupés dans le tableau 5.

\section{DISCUSSION ET CONCLUSIONS}

Une vue panoramique sommaire de la composition des huiles de citronella acclimatés en Afrique centrale et occidentale, au Brésil et en Amérique 
Tableau 2. Variation stationnelle de la teneur (\%) et composition chimique (\%) des huiles essentielles de quelques échantillons de Cymbopogon nardus - Stational change yields (\% DM) and chemical composition (\%) of essential oils of some samples of Cymbopogon nardus.

\begin{tabular}{lcccccccc}
\hline Paramètre & \multicolumn{2}{l}{ Échantillons } \\
\cline { 2 - 9 } & CN12/15 & CN13/15 & CN16/15 & CN19/15 & CN20/15 & CN17/15 & Moyenne & Coeff. Var. (\%) \\
\hline Localisation & SSc & SSc & LKc & LKv & LKv & NKp & & \\
\hline Teneur en HE & 2,0 & 1,8 & 1,8 & 1,8 & 1,7 & 2,0 & 1,9 & 8 \\
Limonène & 3,1 & 3,4 & 3,2 & 2,7 & 2,8 & 2,9 & 3,0 & 10 \\
$\sum$ isopulégols & 1,6 & 1,6 & 2,0 & 1,4 & 1,4 & 2,2 & 1,7 & 18 \\
Citronellal & 44,9 & 44,4 & 47,1 & 41,1 & 45,0 & 45,4 & 44,7 & 4 \\
Citronellol & 11,9 & 11,1 & 11,5 & 11,1 & 10,3 & 12,0 & 11,3 & 5 \\
Géraniol & 22,4 & 22,9 & 21,0 & 25,7 & 23,5 & 21,5 & 22,8 & 7 \\
Acétate de citronellyle & 1,8 & 1,4 & 1,1 & 1,3 & 1,5 & 1,2 & 1,4 & 14 \\
Acétate de géranyle & 1,9 & 1,5 & 0,9 & 1,7 & 2,1 & 1,1 & 1,5 & 33 \\
Béta élémène & 1,8 & 1,6 & 1,8 & 1,7 & 1,5 & 1,9 & 1,7 & 6 \\
Germacrène & 1,3 & 1,2 & 1,4 & 1,6 & 1,5 & 1,4 & 1,4 & 7 \\
Delta cadinène & 1,5 & 1,5 & 1,5 & 1,6 & 1,4 & 1,7 & 1,5 & 7 \\
Élémol & 2,2 & 2,9 & 2,5 & 3,0 & 2,5 & 2,6 & 2,6 & 12 \\
TOTAL (majeurs) & $\mathbf{9 6 , 4}$ & $\mathbf{9 5 , 3}$ & $\mathbf{9 5 , 8}$ & $\mathbf{9 4 , 7}$ & $\mathbf{9 5 , 2}$ & $\mathbf{9 5 , 9}$ & & \\
\hline
\end{tabular}

SSc : colline Sésé - Sésé Hill ; LKc : colline Loukoko - Loukoko Hill ; LKv : vallée Loukoko - Loukoko valley ; NKp : plateau Nkama - Nkama plateau.
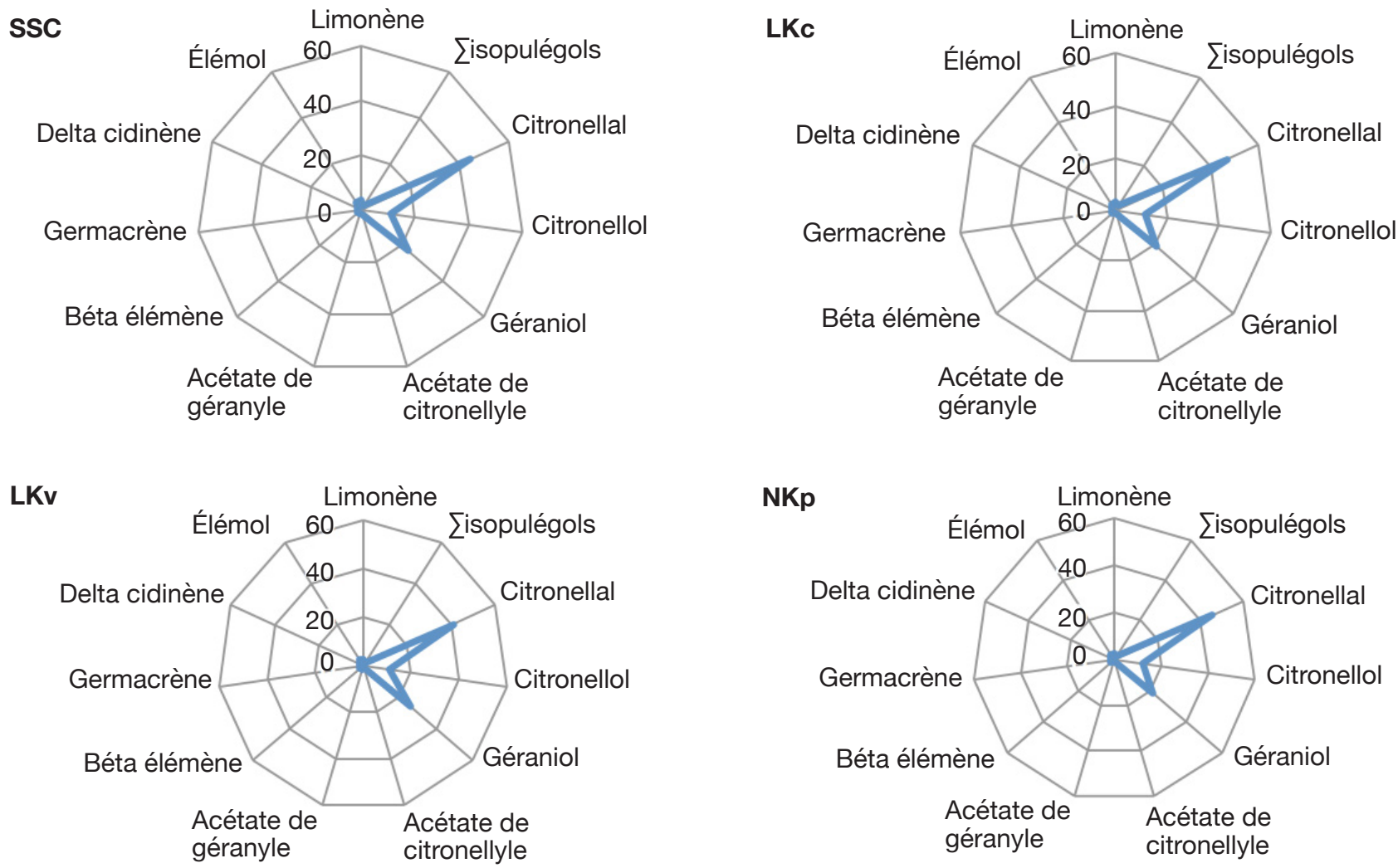

Figure 3. Variation, en fonction des stations de culture, de la composition des huiles essentielles de Cymbopogon nardus acclimaté sur le Plateau des Cataractes (représentation radar-plots) - Stational change of essential oils composition of Cymbopogon nardus acclimatized in the Plateau des Cataractes (radar-plots).

SSC, LKc, LKv, NKp : voir tableau 2 - see table 2. 


\section{Constituants majeurs}

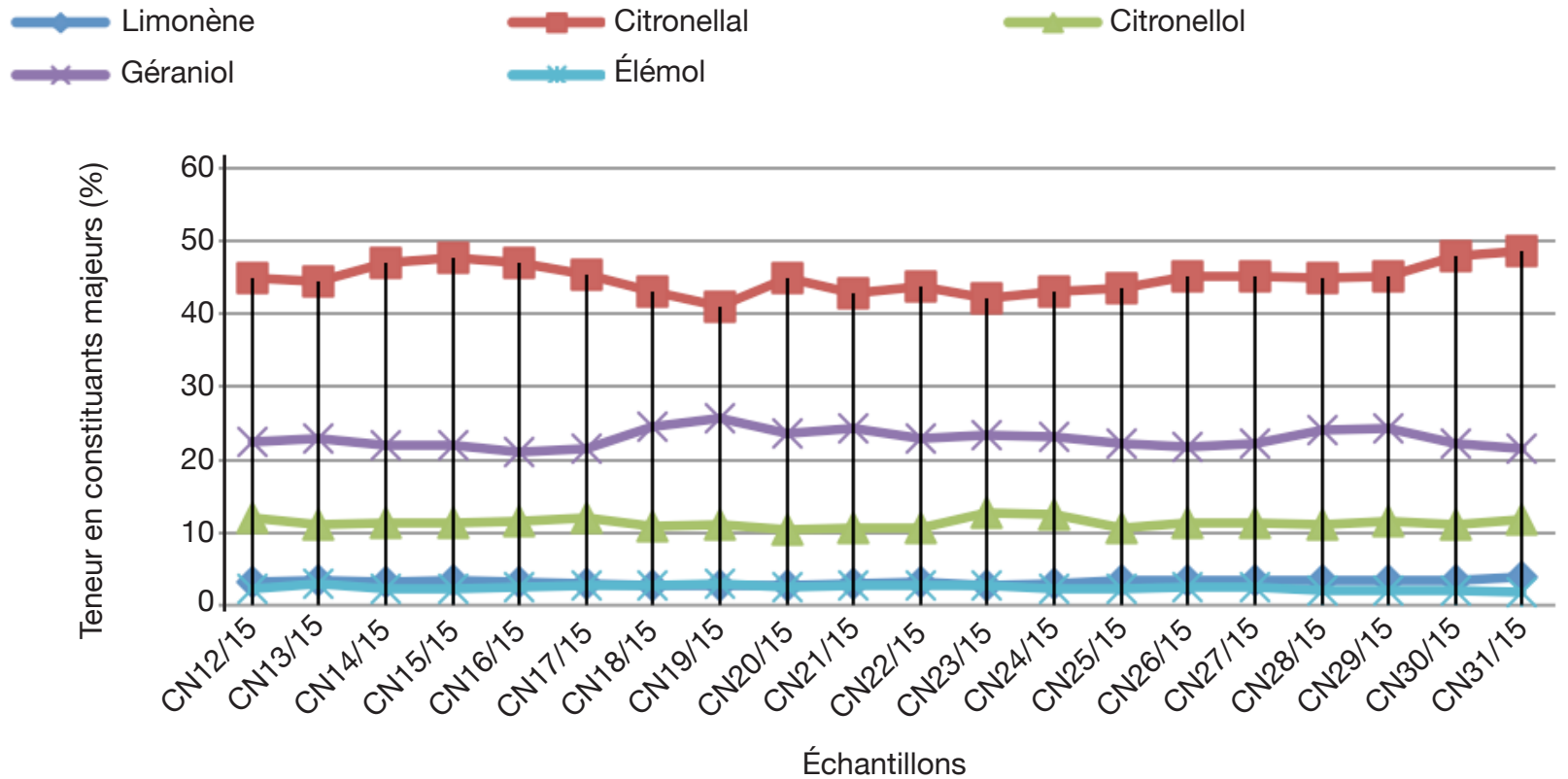

\section{Constituants mineurs}

\begin{tabular}{|c|c|c|}
\hline Kisopulégols & _ Acétate de citronellyle & Acétate de géranyle \\
\hline Béta élémène & $\ldots$ Germacrène D & - Germacrène A \\
\hline
\end{tabular}

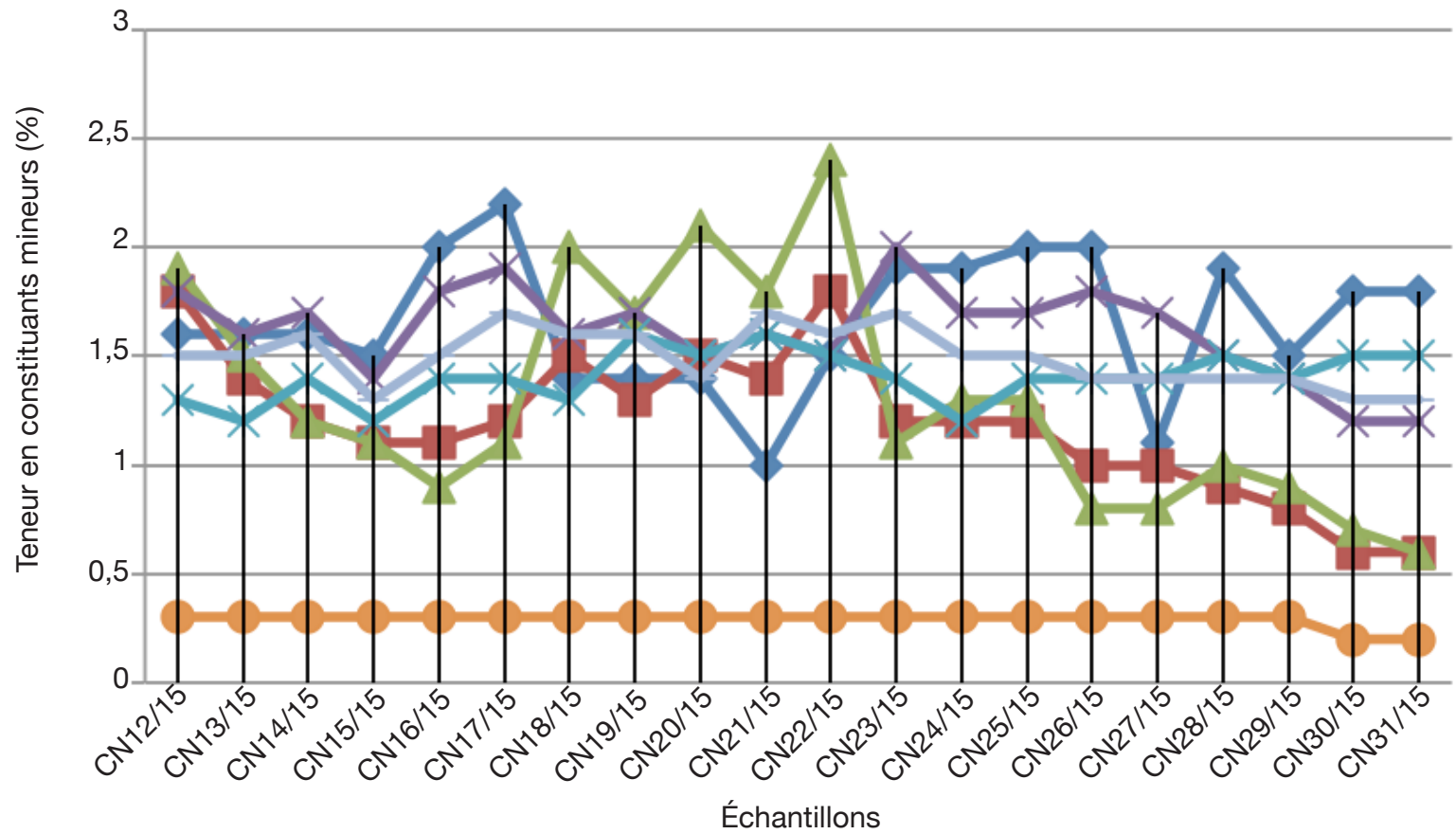

Figure 4. Variation de la composition chimique des huiles essentielles de Cymbopogon nardus (campagne 2015) - Change in the chemical composition of essential oils of Cymbopogon nardus (crop year: 2015). 
<smiles>CC(C)=CCCC(C)C</smiles>

Néral<smiles>C=CC(C)(O)CCC=C(C)C</smiles>

Linalol<smiles>CC(C)=CCCC(C)=CC=O</smiles>

Géranial<smiles>CC(C)=CCCC(C)=CCCO</smiles>

Géraniol<smiles>CC(C)=CCCC(C)CC=O</smiles>

Citronellal<smiles>CC(C)=CCCC(C)CCO</smiles>

Citronellol

Figure 5. Formules chimiques des principaux constituants des huiles essentielles de citronella - Chemical formulas of the main constituents of citronella essential oils.

Figure 6. Cercle de corrélation des variables (a) et positionnement des individus (b) sur le premier plan principal F1F2 (70 \% d'information) - Circle of correlation of the variables $(\boldsymbol{a})$ and positioning of individuals $(\boldsymbol{b})$ on the first principal plane F1F2 (70\% information).
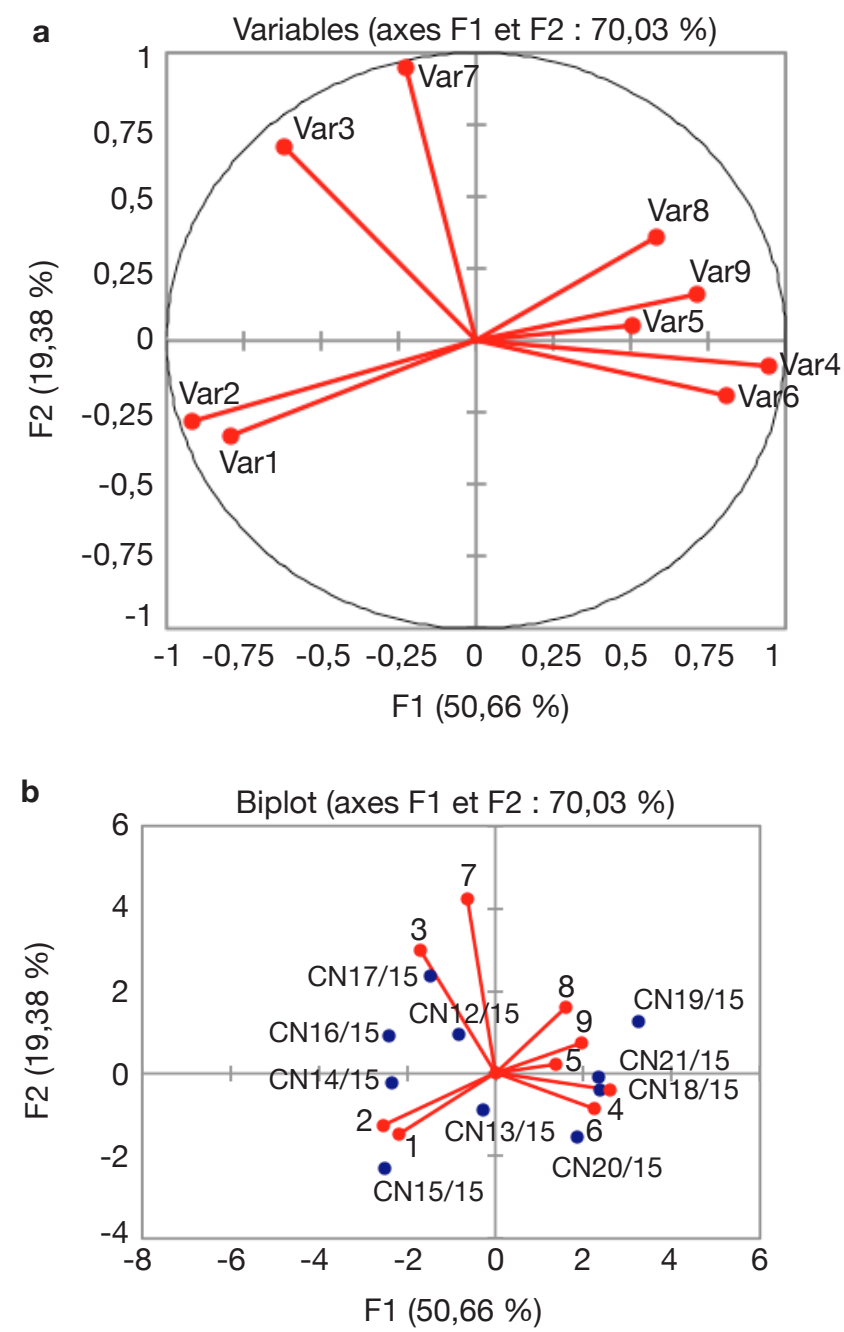

Tableau 3. Composition en constituants majeurs des huiles essentielles de Cymbopogon nardus produites en 2015 - Major constituent composition of Cymbopogon nardus essential oils produced in 2015.

\begin{tabular}{llllllllll}
\hline Échantillon & $\mathbf{1}$ & $\mathbf{2}$ & $\mathbf{3}$ & $\mathbf{4}$ & $\mathbf{5}$ & $\mathbf{6}$ & $\mathbf{7}$ & $\mathbf{8}$ & $\mathbf{9}$ \\
\hline CN12/15 & 3,1 & 44,9 & 11,9 & 22,4 & 1,8 & 1,9 & 1,8 & 1,3 & 2,2 \\
CN13/15 & 3,4 & 44,4 & 11,1 & 22,9 & 1,4 & 1,5 & 1,6 & 1,2 & 2,9 \\
CN14/15 & 3,2 & 47,0 & 11,2 & 22,0 & 0,2 & 1,2 & 1,7 & 1,4 & 2,3 \\
CN15/15 & 3,4 & 47,8 & 11,2 & 22,1 & 1,1 & 1,1 & 1,4 & 1,2 & 2,3 \\
CN16/15 & 3,2 & 47,1 & 11,5 & 21,0 & 1,1 & 0,9 & 1,8 & 1,4 & 2,5 \\
CN17/15 & 2,9 & 45,4 & 12,0 & 21,5 & 1,2 & 1,1 & 1,9 & 1,4 \\
CN18/15 & 2,6 & 43,1 & 10,8 & 24,5 & 1,5 & 2,0 & 1,6 & 1,3 \\
CN19/15 & 2,7 & 41,1 & 11,1 & 25,7 & 1,3 & 1,7 & 1,7 & 1,6 \\
CN20/15 & 2,8 & 45,0 & 10,3 & 23,5 & 1,5 & 2,1 & 1,5 & 1,5 \\
CN21/15 & 2,9 & 42,9 & 10,7 & 24,3 & 1,4 & 1,8 & 1,6 & 3,0 \\
Moyenne & 3,0 & 44,9 & 11,2 & 23,0 & 1,3 & 1,5 & 1,7 & 2,5 \\
Écart-type & 0,3 & 2,1 & 0,5 & 1,5 & 0,4 & 0,4 & 0,2 & 1,4 & 0,1 \\
\hline
\end{tabular}

1 : limonène $; 2$ : citronellal ; 3 : citronellol ; 4 : géraniol ; 5 : acétate de citronellyle ; $6:$ acétate de géranyle ; $7:$ béta élémène ; 8 : germacrène $\mathrm{D} ; 9$ : élémol. 
Tableau 4. Matrice de corrélation de neuf constituants de Cymbopogon nardus en ACP - Correlation matrix of nine Cymbopogon nardus constituents (PCA).

\begin{tabular}{llllllllll}
\hline Var* & $\mathbf{1}$ & $\mathbf{2}$ & $\mathbf{3}$ & $\mathbf{4}$ & $\mathbf{5}$ & $\mathbf{6}$ & $\mathbf{7}$ & $\mathbf{8}$ & $\mathbf{9}$ \\
\hline 1 & 1 & 0,7145 & 0,3273 & $-0,6749$ & $-0,3337$ & $-0,6199$ & $-0,1361$ & $-0,6198$ & $-0,4528$ \\
2 & 0,7145 & 1 & 0,3148 & $-0,8991$ & $-0,4953$ & $-0,6762$ & $-0,0533$ & $-0,5295$ & $-0,7946$ \\
3 & 0,3273 & 0,3148 & 1 & $-0,5981$ & $-0,0601$ & $-0,5565$ & 0,7516 & $-0,3258$ & $-0,3628$ \\
4 & $-0,6749$ & $-0,8991$ & $-0,5981$ & 1 & 0,3595 & 0,7309 & $-0,3295$ & 0,4944 & 0,6936 \\
5 & $-0,3337$ & $-0,4953$ & $-0,0601$ & 0,3595 & 1 & 0,6300 & $-0,0521$ & $-0,0271$ & 0,2599 \\
6 & $-0,6199$ & $-0,6762$ & $-0,5565$ & 0,7309 & 0,6300 & 1 & $-0,2886$ & 0,2731 & 0,2739 \\
7 & $-0,1361$ & $-0,0533$ & 0,7516 & $-0,3295$ & $-0,0521$ & $-0,2886$ & 1 & 0,1833 & $-0,0373$ \\
8 & $-0,6198$ & $-0,5295$ & $-0,3258$ & 0,4944 & $-0,0271$ & 0,2731 & 0,1833 & 1 & 0,3575 \\
9 & $-0,4528$ & $-0,7946$ & $-0,3628$ & 0,6936 & 0,2599 & 0,2739 & $-0,0373$ & 0,3575 & 1 \\
\hline
\end{tabular}

* 1 - 9 : voir tableau 3 - see table 3 .

Tableau 5. Variation pluri-annuelle de la composition chimique moyenne (\% \pm SD) en constituants majeurs des huiles essentielles de Cymbopogon nardus acclimaté sur le Plateau des Cataractes (2003-2016) - Multi-annual variation in average chemical composition $(\% \pm S D)$ of major constituents of the essential oils of Cymbopogon nardus acclimatized in the Plateau des Cataractes (2003-2016).

\begin{tabular}{|c|c|c|c|c|c|c|}
\hline \multirow[t]{2}{*}{ Constituant } & & \multicolumn{5}{|c|}{ Année (nombre d'échantillons analysés) } \\
\hline & & $2003(n=10)$ & $2012(n=10)$ & $2013(n=7)$ & $2015(n=11)$ & $2016(n=14)$ \\
\hline Limonène & 1 & $2,64(1,2)$ & $2,39(1,0)$ & $2,18(0,17)$ & $3,02(0,3)$ & $2,99(0,2)$ \\
\hline Citronellal & 2 & $39,04(9,1)$ & $44,14(2,6)$ & $33,68(4,78)$ & $44,87(2,1)$ & $43,91(3,3)$ \\
\hline Citronellol & 3 & $10,56(3,3)$ & $12,16(0,8)$ & $8,08(1,01)$ & $11,18(0,5)$ & $10,24(0,8)$ \\
\hline Géraniol & 4 & $27,28(5,1)$ & $23,39(1,8)$ & $20,65(1,71)$ & $22,99(1,5)$ & $20,58(5,8)$ \\
\hline Acétate de citronellyle & 5 & $1,73(1,0)$ & $0,76(0,4)$ & $2,37(0,52)$ & $1,25(0,4)$ & $1,12(0,3)$ \\
\hline Acétate de géranyle & 6 & $3,49(2,8)$ & $0,88(0,7)$ & $3,20(1,48)$ & $1,53(0,4)$ & $1,52(0,6)$ \\
\hline Béta élémène & 7 & $0,32(0,3)$ & $1,18(0,3)$ & $2,82(0,35)$ & $1,66(0,2)$ & $1,45(0,2)$ \\
\hline Germacrène D & 8 & $0,86(0,9)$ & $0,61(0,3)$ & $2,70(0,37)$ & $1,39(0,1)$ & $1,18(0,2)$ \\
\hline Élémol & 9 & $2,81(1,4)$ & $3,56(0,8)$ & $7,97(2,72)$ & $1,39(0,1)$ & $2,28(0,4)$ \\
\hline
\end{tabular}

Tableau 6. Aperçu sommaire de la composition (\%) des huiles essentielles de citronella introduits en Afrique et au Brésil - Summary overview of the essential oils composition (\%) of citronella introduced in Africa and Brazil.

\begin{tabular}{lllcll}
\hline Pays & Citronellal & Géraniol & Citronellol & Total & Référence \\
\hline Ghana $1^{*}$ & 46,90 & 19,80 & 13,10 & 79,80 & Koumaglo, 2004 \\
\hline Ghana 2** & 19,40 & 41,40 & 11,60 & 72,40 & Koumaglo, 2004 \\
\hline Togo1* & 48,00 & 18,60 & 6,80 & 73,40 & Koumaglo, 2004 \\
\hline Togo 2* & 30,58 & 23,93 & 7,70 & 62,21 & Nyamador et al, 2010 \\
\hline Bénin 1* & 47,80 & 25,00 & 10,30 & 83,10 & Koumaglo, 2004 \\
\hline Bénin 2** & 28,40 & 34,50 & 11,50 & 74,40 & Nombault, 2015 \\
Côte d'Ivoire*** & 29,20 & 29,30 & 12,70 & 71,20 & Doumbia et al., 2014 \\
Brésil* & 34,61 & 23,18 & 12,10 & 68,89 & Oliveira et al., 2011 \\
Congo 1* & 44,87 & 22,99 & 11,18 & 79,04 & Présent travail \\
Congo 2*** & 32,55 & 30,10 & 6,44 & 69,09 & Loubaki, 2003 \\
\hline
\end{tabular}

$*: \%$ citronellal $>\%$ geraniol (type Java) $; * *: \%$ geraniol $>\%$ citronellal (type Ceylan) $; * * *: \%$ citronellal $\%$ geraniol. 
latine (Tableau 6) indique clairement que les deux chémotypes de citronella (Java et Ceylan) ont été diffusés à travers le monde, parfois sous l'appellation unique de Cymbopogon nardus. La composition de l'essence de citronella de type Java est très proche de celle de Cymbopogon winterianus, alors que celle de l'essence de Cymbopogon nardus var. 'nardus' correspond au citronella type Ceylan.

Les deux chémotypes sont présents au Ghana, au Togo et au Bénin sous l'appellation Cymbopogon nardus : type Java ou type Ceylan.

Les éclats introduits au Congo correspondent au Cymbopogon nardus type Java du Bénin et du Togo (Koumaglo, 2014) et donc proches de Cymbopogon winterianus.

L'incertitude sur la composition de ces deux espèces se rencontre même dans le Sud-Est asiatique où elles sont endémiques, compte tenu de la variabilité élevée des espèces et variétés regroupées sous l'appellation citronella (Tableau 7).

On constate d'après ce tableau que l'huile produite sur le Plateau des Cataractes est plus proche de celle produite par C.winterianus que celle de C.nardus découlant de la compilation de la littérature par Akhila (2010).

On arrive même à des teneurs en citronellal $(32,55 \%)$ et en géraniol $(30,10)$ quasi-identiques (Tableau 6).

Par ailleurs, même au sein de C.nardus, il y a certaines anomalies qui méritent d'être relevées: les feuilles jeunes de C.nardus produisent une huile proche de C.nardus type Ceylan, alors que les feuilles adultes donnent une huile semblable à celle du type Java, proche donc de $C$. winterianus.

Il ressort clairement de cette étude que la production del'huile decitronnellepar lescommunautés villageoises doit être encadrée par une analyse systématique, tout au moins au lancement des exploitations, afin d'identifier lequel des deux types d'huiles essentielles est produit par le matériel végétal formellement identifié à C. nardus sur le plan morphologique.

Tableau 7. Aperçu sommaire de la composition (\%) des huiles essentielles de citronella en Asie du sud-est et au CongoBrazzaville - Summary overview of the essential oil composition (\%) of citronella in Southeast Asia and Congo-Brazzaville.

\begin{tabular}{|c|c|c|c|c|c|c|}
\hline & \multicolumn{6}{|c|}{ Référence } \\
\hline & \multirow[t]{2}{*}{ CN1 } & \multirow[t]{2}{*}{ CNCongo } & \multirow[t]{2}{*}{$\mathbf{C W}$} & \multicolumn{2}{|c|}{ CN2 (feuilles) } & \multirow[t]{2}{*}{ CN3 } \\
\hline & & & & jeunes & adultes & \\
\hline $\mathrm{HE}$ & - & & - & $0,4-0,7$ & - & \\
\hline Limonène & $9-28$ & 3,18 & - & - & - & 1,0 \\
\hline Linalol & 8 & 0,67 & - & - & - & - \\
\hline$\sum$ isopulégols & - & 2,28 & - & - & - & - \\
\hline Citronellal & $5-16$ & 47,16 & $30-45$ & 39,66 & 4,80 & - \\
\hline Citronellol & $8-20$ & 11,98 & $4-10$ & 12,98 & 6,69 & - \\
\hline Citral & 1,17 & 1,17 & - & - & 26,61 & 0,9 \\
\hline Géraniol & $20-30$ & 22,32 & $20-25$ & 18,83 & 46,10 & 75,1 \\
\hline Acétate de citronellyle & traces & 0,66 & 3 & - & - & - \\
\hline Eugénol & traces & 0,68 & traces & - & - & - \\
\hline Acétate de géranyle & $7-8$ & 0,73 & 4 & - & - & 7,6 \\
\hline Béta élémène & - & 1,19 & - & - & - & traces \\
\hline Germacrène D & - & 0,62 & - & - & - & - \\
\hline Delta cadinène & - & 0,98 & - & - & - & 0,2 \\
\hline Élémol & - & 3,00 & - & - & - & - \\
\hline Germacrène D 4 ol & - & 0,61 & - & - & - & - \\
\hline Alpha cadinol & - & 0,68 & - & - & - & - \\
\hline
\end{tabular}

CN1 : Cymbopogon nardus, compilation Akhila (2010); CNCongo : Cymbopogon nardus, présent travail - present work; CW : Cymbopogon winterianus, compilation Akhila (2010) ; CN2 : Cymbopogon nardus Malaisie (Chong Jun Weng et al., 2015) ; CN3 : Cymbopogon nardus var. 'confertiflorus'. 


\section{Bibliographie}

Adams R.P., 2012. Identification of essential oil components by gas chromatography/mass spectroscopy. Carol Stream, IL, USA: Allured Publishing Corporation.

Akhila A., 2010. Chemistry and biogenesis of essential oils from the genus Cymbopogon. In: Akhila A., ed. Essential oil-bearing grasses: the genus Cymbopogon. Boca Raton, FL, USA: CRC Press, 25-106.

Anonyme, 2010. Développement des filières de commercialisation d'huiles essentielles d'E. citriodora, produit forestier non ligneux à forte valeur ajoutée, par les communautés villageoises du Congo. Projet CFC/ ITTO/80/PD364/05 Rev.4(I), http://www.itto.int/files/ itto_project_db_input/2582/Project/F-PD-364-05-R4-IRep-of-Congo-Project-Document.pdf, (5/5/2017).

Chong Jun Weng D., Latip J., Hasbullah S.A. \& Sastrohamidjojo H., 2015. Optimal extraction and evaluation on the oil content of citronella oil from Cymbopogon nardus. Malaysian J. Anal. Sci., 19(1) 7176.

Chowdhury J.U. et al., 1998. Studies on the essential oil bearing plants of Bangladesh. Part IV. Composition of the leaf oil of three Cymbopogon species: C. flexuosus (Nees ex Steud.) Wats., C.nardus (L.) Rendle var. confertiflorus (Steud.) N.L. Bor and C. martinii (Roxb.) Wats var. martinii. J. Essent. Oil Res., 10, 301-306.

Doumbia M. et al., 2014. Toxicity of Cymbopogon nardus (Poaceae) against stored food insect pests. Int. J. Farming Allied Sci., 3(8), 903-909.

Ganjewala D., 2009. Cymbopogon essential oils : chemical compositions and bioactivities. Int. J. Essent. Oil Ther., 3, 56-65.

König W.A.,Hochmuth D.H.\& Joulain D.,2001.Terpenoids and related constituents of essential oils. Library of Mass Finder 2.1. Hamburg, Germany: University of Hamburg, Institute of Organic Chemistry.

Koumaglo H.K., 2004. Production de l'essence de citronnelle par les coopératives villageoises en Afrique occidentale. In : Collin G. \& Garneau F.X., eds. Les huiles essentielles : de la plante à la commercialisation, manuel pratique. Québec, Canada: Université de Chicoutimi, 81-97.
Loubaki L., 2003. Contribution à l'optimisation de la production et de l'extraction des huiles essentielles de Cymbopogon citratus et de Cymbopogon nardus en culture paysanne. Thèse de doctorat : Université Marien Ngouabi, Brazzaville (Congo).

NIST (National Institute of Standards and Technology), 1999. PC version 1.7 of the NIST/EPA/NIH Mass Spectal library. Perking-Elmer Norwalk, CT, NIS Chemistry WebBook NIST Standard. Gaitherburg, MD, USA: NIST.

Nombault Nienzy Praudhyge Jaynereuse, 2015. Suivi de la production qualitative de l'huile essentielle de Cymbopogon nardus par la réaction d'oximation. Mémoire de master: Université Marien Ngouabi, Brazzaville (Congo).

Nyamador S.W., Ketoh G.K.,Koumaglo H.K. \& Glitho I.A., 2010. Activités ovicide et larvicide des huiles essentielles de Cymbopogon gigateus Chiov. et Cymbopogon nardus L. Rendle sur les stades immatures de Callosobruchus maculatus F. et Callosobruchus subinnotatus Pic. (Coleoptera : Bruchidae). J. Soc. Ouest Afr. Chim., 29, 67-79.

Oliveira M.M.M. et al., 2011. Yield, chemical composition and antilisterial activity of essential oils from Cymbopogon species. Rev. Bras. Plant. Med., 13(1), 8-16.

Silou T. et al., 2017a. Aromatic plants from Plateau des Cataractes (Congo Basin). II. Chemical composition of leaf essential oils of Cymbopogon flexuosus (Nees ex Steud.) Wats var. flexuosus acclimatized in CongoBrazzaville. Adv. J. Food Sci. Technol. (sous presse).

Silou T. et al., 2017b. Aromatic plants from Plateau des Cataractes (Congo Basin). V. Chemical composition of inflorescence essential oils from Cymbopogon flexuosus (Nees ex Steud.) Wats var. ablescens acclimatized in Congo-Brazzaville. J. Food Sci. Technol. (sous presse).

Silou T. et al., 2017c. Plantes aromatiques du Plateau des Cataractes (Bassin du Congo). I. Caractérisation des huiles essentielles des espèces du genre Cymbopogon acclimatées au Congo-Brazzaville. Congo Sci., 5(1), 12-23.

(17 réf.) 